\title{
The ring that resounds on ultrasound.
}

Ankita Shrivastav, Vikram Vinayak Koundanya*, Shalini Singh, Chanda Gupta, Sumit Kumar

Vitreoretina Services, Dr. Shroff's Charity Eye Hospital, New Delhi, India

Accepted on September 26, 2018

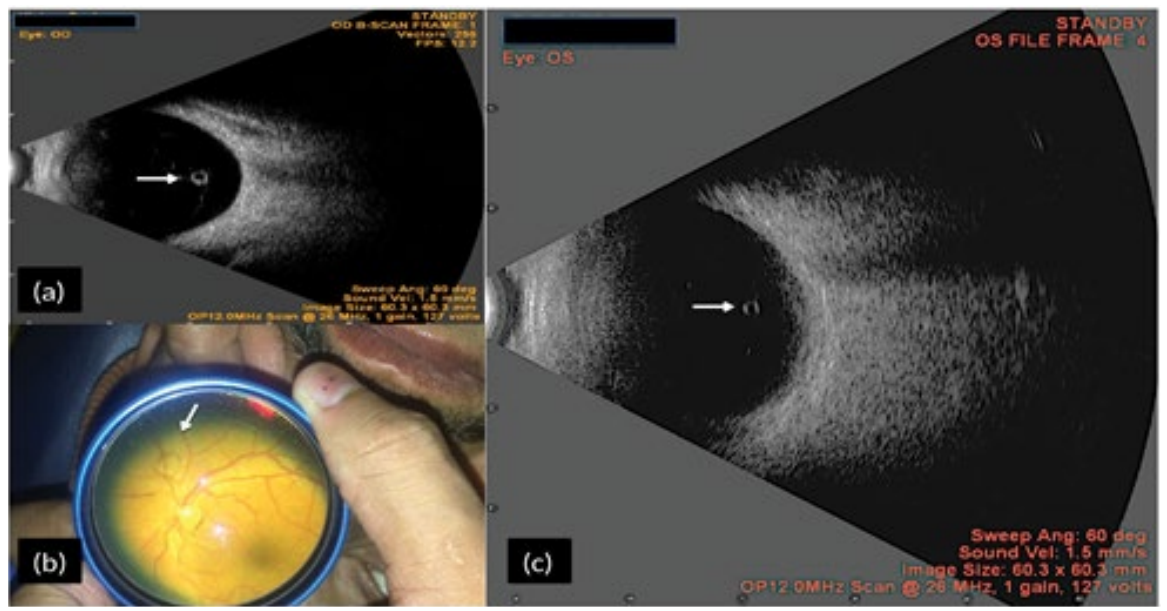

Figure 1 shows B-scan of a 75 year old lady prior to penetrating keratoplasty. A cyst like structure which we presumed to be weiss ring (white arrow) (Figure 1a) was noted. Differentials include pigmented vitreous cyst and parasites like toxoplasma, echinococcus and cysticercosis. To test our hypothesis we performed B-scan on another gentleman in whom weiss ring was seen clinically (Figure 1b). A similar picture appeared on ultrasound (Figure 1c). Fundus examination post operatively confirmed our hypothesis that it was indeed the weiss ring. We hereby propose that Weiss ring should be included in differentials for cystic lesion on ultrasound.

\section{*Correspondence to:}

Vikram Vinayak Koundanya

Vitreoretina Services

Dr. Shroff's Charity Eye Hospital

Kedarnath Road, New Delhi

India

Tel: +91-9500135421

E-mail: vikramkoundanya@gmail.com 